\section{Cabergolina como Alternativa no Tratamento Clínico de Prolactinomas. Experiência na Intolerância/Resistência à Bromocriptina}

\section{RESUMO}

Cabergolina ( $C A B$, Pharmacia) é um agonista dopaminérgico derivado do ergot com longa ação após administração oral, que já tem demonstrado ser de utilidade para o tratamento da hiperprolactinemia. Quarenta e cinco pacientes ( 36 mulheres, 9 homens) com prolactinomas (27 micro, 18 macro), intolerantes e/ou resistentes à bromocriptina (BRC) foram tratados com dose semanal de $C A B$ de 0,25 a $7 \mathrm{mg}$ (mediana: $1 \mathrm{mg}$ ) dividida de 1 a 7 administrações. $O$ tratamento, em compassionate basis, variou de 1 a 38 meses (mediana: 12 meses). Entre os 38 pacientes com intolerância persistente à BRC (sintomas digestivos, $n=27$; hipotensão postural, $n=13$; congestão nasal, $n=5$; manifestações psiquiátricas, $n=4$; retenção urinária, $n=1$ ), somente 5 permaneceram intolerantes à $C A B$ (sintomas digestivos, $n=2$; hipotensão postural, $n=2$; congestão nasal, $n=1$ ). Todos aqueles que toleraram bem a BRC também o fizeram com CAB. Onze casos alcançaram normalização da prolactina durante o uso de BRC. Estes pacientes e outros 19 também resolveram a hiperprolactinemia com CAB. Entretanto, somente 3 dos 7 pacientes resistentes (dose diária de $B R C$ de 10 a $25 \mathrm{mg}$ ) tiveram a prolactina sérica normalizada durante 0 uso de $C A B$. Redução tumoral foi documentada por ressonância magnética ou tomografia computadorizada em 7 macroprolactinomas durante o tratamento com CAB. Assim, devido à sua excelente eficácia, tolerabilidade e comodidade posológica, a CAB apresenta-se como importante alternativa no tratamento clínico dos prolactinomas. (Arq Bras Endocrinol Metab 2000;44/2: 139-43)

Unitermos: Prolactinomas; Prolactina; Drogas dopaminérgicas; Tumores hipofisários; Bromocriptina; Cabergolina.

\begin{abstract}
Cabergoline ( $C A B$, Pharmacia) is a long-acting ergoline dopaminergic agonist for oral administration, already described as a useful drug for hyperprolactinemia therapy. Forty-five patients ( 36 women, 9 men) with prolactinomas (27 micro, 18 macro) with persistent intolerance and/or resistance to bromocriptine (BRC) were treated with a weekly $C A B$ dose of 0.25-7.0mg (median: $1 \mathrm{mg}$ ) divided in one to seven administrations along the week. The treatment, in compassionate basis, lasted from 1 to 38 months (median: $12 \mathrm{mo}$ ). Among the 38 BRC intolerant patients (digestive symptoms, $n=27$; orthostatic hypotension, $n=13$; nasal stuffiness, $n=5$; psychiatric manifestations, $n=4$; urinary retention, $n=1$ ) only 5 remained persistently intolerant to $C A B$ (digestive symptoms, $n=2$; arterial hypotension. $n=2$; nasal stuffiness, $n=1$ ). All BRC tolerant patients accepted CAB well. Eleven cases had serum prolactin levels normalized with BRC. These patients and other 19 also reached normal prolactin levels with $\mathrm{CAB}$. However, only 3 out of the 7 BRC resistant patients (daily dose: 10-25mg) had their serum prolactin levels normalized with CAB. Tumor shrinkage was documented by magnetic resonance imaging or computed tomography scanning in 7 macroprolactinoma patients during $C A B$ treatment. Thus, due to its excellent efficacy, tolerability and low frequency administration.
\end{abstract}

\section{artigo original}

\author{
Nina R.C. Musolino \\ Malebranche B. Cunba Neto \\ Marcello D. Bronstein
}

Unidade de Neuroendocrinologia, Divisão de Neurocirurgia Funcional do Instituto de Psiquiatria, Hospital das Clinicas, Faculdade de Medicina da Universidade de São Paulo, São Paulo, SP.
Recebido em 27/09/1999

Revisado em 01/02/2000 Aceito em 05/02/2000 
$C A B$ can be very useful for medical management of prolactinomas. (Arq Bras Endocrinol Metab 2000;44/2: 139-43)

Keywords: Prolactinoma; Prolactin; Dopaminergic drugs: Pituitary tumors; Bromocriptine; Cabergoline.

O EMPREgO DE AGONISTAS dopaminérgicos no tratamento primário dos prolactinomas já é consagrado. Dentre as drogas dopaminérgicas, a bromocriptina (BRC) é a medicação mais utilizada em todo o mundo $\mathrm{c} o$ acompanhamento de pacientes a longo prazo com esta medicação tem comprovado sua eficácia tanto na reversão das alterações gonadais ligadas à hiperprolactinemia como na redução dos adenomas hipofisários (1). Mesmo o tratamento de gestantes portadoras de prolactinomas com a BRC já é aceito como seguro e efetivo na maioria dos casos (2-4). No entanto, cerca de 5 a $18 \%$ dos pacientes são considerados resistentes à $\mathrm{BRC}$, ou porque não apresentam redução tumoral ou porque não atingem a reversão das alterações hormonais dependentes da hiperprolactinemia (5). Além disso, a intolerância persistente à BRC leva à interrupção do tratamento em outros $10 \%$ dos pacientes, aproximadamente (I). Novos agonistas dopaminérgicos têm sido desenvolvidos nos últimos anos, dentre eles, a cabergolina (CAB), administrada por via oral e com longa ação, tem mostrado melhor tolerância e eficácia quando comparada à BRC (6).

Avaliamos neste estudo a resposta ao tratamento com $\mathrm{CAB} \mathrm{em} 45$ pacientes considerados intolerantes ou resistentes à $\mathrm{BRC}$.

\section{PACIENTES E MÉTODOS}

Quarenta e cinco pacientes, 36 mulheres ( 16 a 56 anos, mediana $=30$ ) e 9 homens ( 25 a 52 anos, mediana $=40$ ), portadores de microprolactinomas (MIC) $(n=27$, tabela 1) ou macroprolactinomas (MAC) $(\mathrm{n}=18$, tabela 2), previamente tratados com $\mathrm{BRC}$ receberam $\mathrm{CAB} \mathrm{cm}$ regime de compassionate basis. Em 38 deles o motivo da

Tabela 1 - Dados dos pacientes com microprolactinomas.

\begin{tabular}{|c|c|c|c|c|c|c|c|c|c|c|}
\hline \multirow[t]{2}{*}{ Pacientes } & \multirow[b]{2}{*}{ sexo } & \multirow[b]{2}{*}{$\begin{array}{l}\text { idade } \\
\text { anos }\end{array}$} & \multicolumn{5}{|c|}{ BROMOCRIPTINA } & \multicolumn{3}{|c|}{ CABERGOLINA } \\
\hline & & & $\begin{array}{c}\text { PRL basal } \\
\mathrm{ng} / \mathrm{ml}\end{array}$ & $\begin{array}{c}\text { Dose/d } \\
\text { mg }\end{array}$ & $\begin{array}{c}\mathrm{PRL} \\
\mathrm{ng} / \mathrm{ml}\end{array}$ & $\begin{array}{c}\text { Uso-tempo } \\
\text { meses }\end{array}$ & $\begin{array}{c}\text { PRL basal } \\
\mathrm{ng} / \mathrm{ml}\end{array}$ & $\begin{array}{c}\text { Dose/sem } \\
\text { mg }\end{array}$ & $\begin{array}{l}\mathrm{PRL} \\
\mathrm{ng} / \mathrm{ml}\end{array}$ & $\begin{array}{l}\text { Uso-tempo } \\
\text { meses }\end{array}$ \\
\hline 1 & $\mathrm{~F}$ & 25 & 82 & 0,6 & & 1 & 53 & 0,5 & 11 & 24 \\
\hline 2 & $\mathrm{~F}$ & 32 & 41 & 2,5 & & 12 & 41 & 1 & 6 & 12 \\
\hline 3 & $\mathrm{~F}$ & 42 & 82 & 2,5 & & 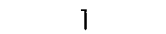 & 72 & 0,5 & 2 & 24 \\
\hline 4 & $\mathrm{~F}$ & 20 & 44 & 2,5 & 54 & 8 & 59 & 0,5 & 31 & 3 \\
\hline 5 & $\mathrm{~F}$ & 27 & 133 & 2.5 & & 12 & 39 & 0.5 & 12 & 4 \\
\hline 6 & $\mathrm{~F}$ & 31 & 130 & 2,5 & 64 & 17 & 60 & 1 & 8 & 4 \\
\hline 7 & $\mathrm{~F}$ & 36 & 114 & 2.5 & & 1 & 114 & 0.5 & 7 & 1 \\
\hline 8 & $\mathrm{~F}$ & 36 & 70 & 2,5 & & 1 & 71 & 0,5 & 20 & 2 \\
\hline 9 & $\mathrm{~F}$ & 40 & 96 & 2,5 & 11 & 1 & 96 & 1.5 & 9 & 8 \\
\hline $10^{\circ}$ & $\mathrm{F}$ & 34 & 54 & 3.7 & 17 & 14 & 64 & 0,25 & 4 & 38 \\
\hline $11^{\mathrm{c}}$ & $\mathrm{F}$ & 30 & 30 & 5 & 6 & 12 & 38 & 1 & 6 & 5 \\
\hline 12 & $\mathrm{~F}$ & 22 & 69 & 5 & 9 & 84 & 39 & 1 & 16 & 12 \\
\hline 13 & $\mathrm{~F}$ & 41 & 75 & 5 & 7 & 84 & & 1 & 3 & 2 \\
\hline 14 & $\mathrm{~F}$ & 16 & 105 & 5 & & 1 & 110 & 0,25 & 3 & 12 \\
\hline 15 & $M$ & 37 & 40 & 5 & & 1 & 40 & 0.5 & 6 & 1 \\
\hline 16 & $\mathrm{~F}$ & 22 & 310 & 5 & 1 & 1 & 173 & 1 & 14 & 15 \\
\hline 17 & $\mathrm{~F}$ & 37 & 85 & 5 & & $i$ & 87 & 1 & 12 & 12 \\
\hline 18 & $\mathrm{~F}$ & 43 & 186 & 7,5 & 152 & 12 & 186 & 1 & 9 & 4 \\
\hline 19 & $\mathrm{~F}$ & 22 & 130 & 10 & 37 & 24 & 105 & 0,5 & 61 & 1 \\
\hline $20^{\mathrm{C}}$ & F & 24 & 60 & 10 & 41 & 96 & 41 & 1 & 6 & 12 \\
\hline 21 & F & 29 & 226 & 10 & 11 & 6 & 81 & 1.5 & 5 & 1 \\
\hline $22 \#$ & $F$ & 28 & 104 & 12,5 & 64 & 9 & 94 & 1,5 & 28 & 5 \\
\hline $23^{c}$ & $M$ & 48 & 408 & 15 & 340 & 12 & 686 & 7 & 220 & 1 \\
\hline $24 \#$ & $F$ & 18 & 519 & 15 & 90 & 6 & 120 & 1,5 & 58 & 1 \\
\hline $25^{*}$ & $\mathrm{~F}$ & 27 & 151 & 15 & 48 & 10 & 149 & 1 & 77 & 1 \\
\hline $26^{* c}$ & $\mathrm{~F}$ & 27 & 162 & 20 & 114 & 120 & 75 & 1,5 & 17 & 13 \\
\hline $27^{*}$ & $F$ & 38 & 93 & 25 & 30 & 12 & 107 & 1,5 & 53 & 2 \\
\hline
\end{tabular}

\footnotetext{
* sem intolerância à BRC

" com intolerância à CAB

c cirurgia prévia
} 
Tabela 2 - Dados dos pacientes com macroprolactinomas.

\begin{tabular}{|c|c|c|c|c|c|c|c|c|c|c|}
\hline \multirow[t]{2}{*}{ Pacientes } & \multirow[b]{2}{*}{ sexo } & \multirow[b]{2}{*}{$\begin{array}{l}\text { idade } \\
\text { anos }\end{array}$} & \multicolumn{5}{|c|}{ BROMOCRIPTINA } & \multicolumn{3}{|c|}{ CABERGOLINA } \\
\hline & & & $\begin{array}{c}\text { PRL basal } \\
\mathrm{ng} / \mathrm{ml}\end{array}$ & $\begin{array}{l}\text { Dose/d } \\
\text { mg }\end{array}$ & $\begin{array}{l}\mathrm{PRL} \\
\mathrm{ng} / \mathrm{ml}\end{array}$ & $\begin{array}{l}\text { Uso-tempo } \\
\text { meses }\end{array}$ & $\begin{array}{c}\text { PRL basal } \\
\mathrm{ng} / \mathrm{ml}\end{array}$ & $\begin{array}{c}\text { Dose/sem } \\
\text { mg }\end{array}$ & $\begin{array}{l}\mathrm{PRL} \\
\mathrm{ng} / \mathrm{ml}\end{array}$ & $\begin{array}{l}\text { Uso-tempo } \\
\text { meses }\end{array}$ \\
\hline $28^{*}$ & $\mathrm{~F}$ & 34 & 132 & 2,5 & 4 & 70 & 49 & 0,5 & 2 & 2 \\
\hline 29 & $\mathrm{~F}$ & 39 & 768 & 2,5 & 370 & 2 & 1580 & 1,5 & 108 & 19 \\
\hline 30 & $M$ & 52 & 360 & 2,5 & & 1 & 368 & 1,5 & 2 & 21 \\
\hline 31 & $\mathrm{~F}$ & 26 & 560 & 3,75 & & 11 & 667 & 1 & 14 & 2 \\
\hline 32 & $\mathrm{~F}$ & 25 & 148 & 5 & 20 & 2 & 95 & 7,5 & 17 & 3 \\
\hline 33 & $M$ & 27 & 204 & 5 & 176 & 11 & 185 & 1,5 & 50 & 1 \\
\hline $34^{*}$ & $\mathrm{~F}$ & 36 & 217 & 5 & 200 & 3 & 199 & 0,5 & 12 & 14 \\
\hline 35 & $\mathrm{~F}$ & 26 & 470 & 5 & 301 & 6 & 332 & 1,5 & 12 & 8 \\
\hline 36 & $\mathrm{~F}$ & 29 & 174 & 7,5 & 19 & 12 & 83 & 1 & 12 & 12 \\
\hline 37 & $M$ & 49 & 354 & 7,5 & & 1 & 551 & 0,5 & 159 & 4 \\
\hline 38 & $\mathrm{~F}$ & 17 & 1616 & 10 & 159 & 6 & 207 & 3,5 & 20 & 15 \\
\hline $39^{\circ}$ & $\mathrm{F}$ & 56 & 353 & 10 & 19 & 1 & 769 & 1,5 & 27 & 12 \\
\hline $40^{c}$ & $\mathrm{~F}$ & 22 & 168 & 15 & 43 & 48 & 212 & 1,5 & 92 & 2 \\
\hline $41^{c}$ & $M$ & 38 & 517 & 20 & 1860 & 20 & 517 & 1,5 & 67 & 1 \\
\hline $42^{*}$ & $M$ & 49 & 1720 & 10 & 248 & 3 & & 3,5 & 9 & 27 \\
\hline $43^{*}$ & $M$ & 38 & 450 & 15 & 138 & 5 & 435 & 3,5 & 19 & 17 \\
\hline $44^{*}$ & $\mathrm{~F}$ & 29 & 201 & 22,5 & 115 & 27 & 106 & 1,5 & 50 & 1 \\
\hline $45^{* c}$ & $M$ & 25 & 1469 & LAR 50 & 233 & 7 & 737 & 1,5 & 370 & 1 \\
\hline
\end{tabular}

* sem intolerância ò $\mathrm{BRC}$

" com intolerância à CAB

c cirurgia prévia

Tabela 3 - Efeitos colaterais apresentados durante o tratamento com bromocriptina e cabergolina.

\begin{tabular}{lcc}
\hline Efeitos colaterais & $\begin{array}{c}\text { Com bromocriptina } \\
(n=38)^{*}\end{array}$ & $\begin{array}{c}\text { Com cabergolina } \\
(n=5)\end{array}$ \\
\hline Sintomas digestivos & 27 & 2 \\
Hipotensão postural & 13 & 2 \\
Congestão nasal & 5 & 1 \\
Manifestações psiquiátricas & 4 & 0 \\
Retenção urinária & 1 & 0 \\
\hline * alguns pacientes apresentaram mais de uma queixa de \\
\multicolumn{2}{l}{ efeitos colaterais. }
\end{tabular}

interrupção do tratamento com BRC foi intolerância persistente a doses diárias de 0,6 a $20 \mathrm{mg}$ (mediana $=5 \mathrm{mg}$, tabela 3 ). Houve normalização da prolactina (PRL) em 11 de 26 pacientes deste grupo que tiveram a PRL dosada durante o uso da BRC (tabelas 1, 2 e 4). Os demais pacientes suspenderam a medicação antes da realização da dosagem devido à intolerância severa. Em 6 pacientes, a PRL sérica não normalizou apesar da boa tolerância à doses de 10 a $25 \mathrm{mg}$ ao dia (mediana $=17,5 \mathrm{mg}$ ) de BRC. Também não alcançou normalização da PRL sérica o único paciente que recebeu BRC injetável, $50 \mathrm{mg}$ a cada 28 dias, com boa tolerância.

A CAB foi utilizada, por via oral, na dose de 0,25 a $7 \mathrm{mg} /$ semana, dividida em $\mathrm{I}$ a 7 administrações. A dose dependeu da tolerância, resposta clínica e laboratorial, além da disponibilidade da medicação.

As dosagens de PRL foram realizadas através de método imunofluorimétrico utilizando kit Delfia Wallac $\mathrm{Oy}$, com valores de referência para mulheres de 2,2 a $18,1 \mathrm{ng} / \mathrm{ml}$ e para homens de 2,0 a $16,1 \mathrm{ng} / \mathrm{ml}$.

Tabela 4 - Distribuição dos 45 pacientes conforme a tolerância e normalização da prolactina sérica durante o tratamento com bromocriptina (BRC) e cabergolina ( $C A B)$.

\begin{tabular}{|c|c|c|c|}
\hline & & $\begin{array}{c}\text { bromocriptina } \\
\text { oral: } 0,6 \text { a } 25 \mathrm{mg} / \mathrm{d}(n=44) \\
\text { LAR: } 50 \mathrm{mg} / \mathrm{m}(n=1)\end{array}$ & $\begin{array}{c}\text { cabergolina } \\
0,25 \text { a } 7 \mathrm{mg} / \mathrm{sem}\end{array}$ \\
\hline Boa tolerância & $\begin{array}{c}\text { SIM } \\
\text { NÃO }\end{array}$ & $\begin{array}{l}7^{*} \\
38\end{array}$ & $\begin{array}{c}40 \\
5\end{array}$ \\
\hline Normalização da PRL ** & $\begin{array}{c}\text { SIM } \\
N \tilde{A} O\end{array}$ & $\begin{array}{l}11 \\
22\end{array}$ & $\begin{array}{l}30 \\
15\end{array}$ \\
\hline
\end{tabular}




\section{RESULTADOS}

Ocorreu normalização dos níveis séricos de PRL cm 30 (67\%) pacientes durante o tratamento com CAB (tabelas 1, 2 e 4). A dose utilizada nesses casos variou de 0,25 a $3,5 \mathrm{mg} /$ semana (mediana $=\mathrm{lmg}$ ), dividida de 1 a 7 administrações. O tempo do tratamento variou de 1 a 38 meses (mediana=12 meses), e foi superior a 3 meses em 22 destes pacientes. Redução tumoral foi observada em todos 7 pacientes com MAC que realizaram ressonância magnética durante o período de tratamento.

Gestação ocorreu em 5 pacientes $(2 \mathrm{com}$ MAC). A CAB foi suspensa em todas as pacientes assim que diagnosticada a gravidez. Não houve nenhum sintoma relacionado a crescimento tumoral. Todos recém-nascidos foram saudáveis, embora parto prematuro tenha ocorrido em duas pacientes.

Efeitos colaterais persistentes ocorreram apenas em 5 dos 38 casos previamente intolerantes à BRC (tabelas 1,2 e 3 ). Estes efeitos indescjáveis foram: enjôo e vômitos em 2, hipotensão postural $\mathrm{cm} 2 \mathrm{e}$ congestão nasal em 1 paciente.

Todos 7 pacientes tolerantes à BRC também aceitaram bem a CAB na dose semanal de 1 a $3,5 \mathrm{mg}$. Destes, três obtiveram normalização da PRL durante $O$ tratamento com $\mathrm{CAB}$ na dose de 1,5 a $3,5 \mathrm{mg} / \mathrm{semana}$. Nestes pacientes, a dose diária de BRC havia sido de 10 a $20 \mathrm{mg}$, sem normalização laboratorial. Nos outros 4 casos a PRL manteve-se elevada durante o uso de 1 a $1,5 \mathrm{mg} /$ semana de CAB. Não sendo a dose aumentada devido a não disponibilidade da medicação.

\section{DISCUSSÃO}

No grupo de pacientes intolerantes à BRC pudemos observar significativa redução dos efeitos colaterais com a CAB. Este resultado está de acordo com a literatura (6-8).

Para analisar o seu efeito em pacientes considerados resistentes à $\mathrm{BRC}$, devemos em primeiro lugar tentar definir o que é resistência. A literatura é controversa, mas a maioria dos autores considera a dose de $15 \mathrm{mg} /$ dia como a dose limite para o diagnóstico de resistência quando a PRL não normaliza $\mathrm{e} / \mathrm{Ou}$ não há redução tumoral (5). Além disso, os mecanismos que levam à resistência também não são claros. $O$ que já está bem estabelecido é que há redução na densidade dos receptores dopaminérgicos $\mathrm{D}_{2}(5)$. Na nossa opinião como na de alguns autores (7) a resistência, em termos práticos, pode ser considerada quando os objetivos do tratamento não são alcançados com a maior dose tolerada pelo paciente. Desse modo, o tratamento por $\mathrm{CAB}$ pode atingir resultados satisfatórios em alguns pacientes que necessitem de doses altas do agonista dopaminérgico mas não tolerem o uso da dose necessária de BRC. Além disso, obtivemos normalização da PRL com CAB e não com BRC em 3 pacientes que toleraram previamente 10,15 c $20 \mathrm{mg}$ diárias de BRC. Colao e cols. (9) mostraram normalização da PRL durante tratamento com $C A B \mathrm{~cm} 23$ de 27 pacientes parcialmente resistentes à dose de $15 \mathrm{mg}$ de BRC. A dose de CAB utilizada por esses autores variou de 0,5 a $3 \mathrm{mg}$ por semana. Essa diferença de resposta pode ser explicada pela maior afinidade e maior tempo de ligação da $\mathrm{CAB}$ aos receptores $\mathrm{D}_{2}(10)$. Além disso, sua meia-vida prolongada e maior tolerabilidade podem ser, ao menos $\mathrm{cm}$ parte, responsáveis por seu maior efeito.

Quanto ao uso de CAB na gestação, apresentamos 5 pacientes que engravidaram durante o uso da medicação sem teratogênese. A literatura já conta com mais de 260 gestações $(8,11,12)$ que ocorreram em pacientes em uso de CAB. No entanto, não há, até o momento, segurança comprovada do seu uso durante a gravidez. Devido à sua meia-vida longa, mesmo com sua suspensão precoce haverá manutenção de seu efeito ainda por maior tempo do que quando a BRC é suspensa. No entanto, o uso da BRC já é bem aceito, mesmo quando mantida por toda a gravidez, sem evidências de complicações (13). É possível que tenhamos o mesmo resultado após alguns anos de experiência com a CAB. Mas, até o momento, devemos utilizar, preferencialmente, a BRC em pacientes que desejem engravidar.

Em conclusão, devido à sua excelente tolerabilidade e comodidade posológica, a CAB apresenta-se como importante alternativa no tratamento clínico dos prolactinomas.

\section{AGRADECIMENTOS}

Agradecemos à Pharmacia \& Upjohn pelo fornecimento da cabergolina (Dostinex ${ }^{\circledR}$ ) para o teste $\mathrm{e}$ tratamento inicial dos pacientes.

\section{REFERÊNCIAS}

1. Bronstein MD. Hiperprolactinemia. In: Wajchenberg BL, editor. Tratado de Endocrinologia Clínica. São Paulo: Roca, 1992: 135-64.

2. Bronstein MD, Musolino NR, Cardim CS, Marino Jr R. Hiperprolactinemia e gravidez: evolução de 52 gestações. Rev Bras Ginecol Obst 1988; 10: 175-8.

3. Weil $C$. The safety of bromocriptine in hyperprolactinaemic female infertility: a literature review. Curr Med Res Opin 1986;10:172-95. 
4. Molitch ME. Pituitary diseases in pregnancy. Semin Periantol 1998:22:457-70.

5. Pellegrini I, Rasolonjanahary R, Gunz $G$, et al. Resistance to bromocriptine in prolactinomas. J Clin Endocrinol Metab 1989;69:500-9.

6. Webster J, Piscitelli $G$, Polli $A$, et al. Cabergoline versus bromocriptine in the treatment of hyperprolactinaemic amenorrhoea. N Engl J Med 1994;331:904-9.

7. Ferrari C, Paracchi A, Mattei AM, et al. Cabergoline in the long-term therapy of hyperprolactinemic disorders. Acta Endocrinol 1992; 126:489-94.

8. Verhelst J. Abs R, Maiter D, et al. Cabergoline in the treatment of hyperprolactinemia: A study in 455 patients. J Clin Endocrinol Metab 1999;84:2518-22.

9. Colao A, Di Sarno A, Sarnacchiaro F, et al. Prolactinomas resistant to standard dopamine agonists respond to chronic cabergoline treatment. J Clin Endocrinol Metab 1997:82:876-83.

10. Biller BMK, Molitch ME, Vance ML, et al. Treatment of prolactin-secreting macroadenoma with once-weekly dopamine agonist cabergoline. J Clin Endocrinol Metab 1996;81:2338-43

11. Robert E, Musatti L, Piscitelli G, Ferrari Cl. Pregnancy outcome after treatment with the ergot derivative, cabergoline. Reprod Toxicol 1996; 10:333-7.

12. Rains CP. Bryson HM, Fitton A. Cabergoline. A review of its pharmacological properties and therapeutic potential in the treatment of hyperprolactinaemia and inhibition of lactation. Drugs 1995;49:255-79

13. De Wit W, Coelingh Bennink HJ, Gerards LJ. Prophylactic bromocriptine treatment during pregnancy in women with macroprolactinomas: report of 13 pregnancies. $\mathbf{B r} \mathbf{J}$ Obstet Gynaecol 1984;91: 1059-69.

\section{Endereço para correspondência:}

Marcello Delano Bronstein

Av. Nove de Julho, 3858

01406-100 São Paulo, SP 